\title{
BUILDING CHANGE DETECTION BY COMBINING LiDAR DATA AND ORTHO IMAGE
}

\author{
Daifeng Peng a, Yongjun Zhang ${ }^{\text {a, * }}$ \\ ${ }^{\text {a }}$ School of Remote Sensing and Information Engineering, Wuhan University, Wuhan, 430079, China \\ (daifeng, zhangyj)@whu.edu.cn
}

Commission III, WG III/4

KEY WORDS: LiDAR data, Ortho Image, DSM, Building, 3D Change Detection

\begin{abstract}
:
The elevation information is not considered in the traditional building change detection methods. This paper presents an algorithm of combining LiDAR data and ortho image for 3D building change detection. The advantages of the proposed approach lie in the fusion of the height and spectral information by thematic segmentation. Furthermore, the proposed method also combines the advantages of pixel-level and object-level change detection by image differencing and object analysis. Firstly, two periods of LiDAR data are filtered and interpolated to generate their corresponding DSMs. Secondly, a binary image of the changed areas is generated by means of differencing and filtering the two DSMs, and then thematic layer is generated and projected onto the DSMs and DOMs. Thirdly, geometric and spectral features of the changed area are calculated, which is followed by decision tree classification for the purpose of extracting the changed building areas. Finally, the statistics of the elevation and area change information as well as the change type of the changed buildings are done for building change analysis. Experimental results show that the completeness and correctness of building change detection are close to $81.8 \%$ and $85.7 \%$ respectively when the building area is larger than $80 \mathrm{~m}^{2}$, which are increased about $10 \%$ when compared with using ortho image alone.
\end{abstract}

\section{INTRODUCTION}

LULC (Land Use and Land Cover) change information can be achieved through the analysis of multi-temporal remote sensing images and geographic information data using change detection methodologies (Singh, 1989; Bovolo and Bruzzone, 2007;

Nutini et al., 2013; Zhu and Woodcock, 2014; Franklin et al., 2015). With the improvement of the resolution of remote sensing images, particular attention is paid on the analysis of special artificial objects, especially the buildings (Champion, 2007). Building change information is of great importance to disaster assessment and post-disaster reconstruction, urban growth monitoring and environmental change researches. Traditional urban change information can be obtained through the analysis of spectral and textual features of aerial or remote sensing images (Niemeyer et al., 2007; Huo et al., 2010; Adar et al., 2014; Cao et al., 2014; Wang et al., 2015). However, the above methods mainly consider the radiation information of images, leading to a large amount of omission and commission errors. Especially for buildings, spectral information differs greatly due to different roof materials and buildings share similar textual features with roads, which makes it difficult for extracting building change information by using spectral and textual information alone. Moreover, both shadows of high-rise buildings and titled buildings caused by perspective projection cause many problems in the building change detection, which also bring challenges to obtain the building change information using the radiation information alone (Knudsen and Olsen, 2003; Vu et al., 2004). Height variation is an important change indicator of artificial buildings. In addition to that, elevation information is of great significance in the exclusion of non-building objects such as trees and in the distinguishing between buildings and roads which share the similar spectral and textual features (Murakami, 1999; Gamba and Houshmand, 2002; Vögtle and Steinle, 2004).

In recent decades, building change detection methods, which take elevation information into account, have made significant progresses. According to different elevation information acquisition modes, those methods can be divided into two categories:

1. DSM is generated by stereo images and building change map is generated by DSM comparison and post-processing. Jung (2004) generated two periods of DSMs based on aerial stereo images, which is followed by DSM comparison and edge features extraction and classification. Tian et al. (2014) carried out 3D building change detection using stereo images and DSMs generated with stereo matching technology. The Dempster-Shafer fusion theory was adopted to implement the joint use of height changes and Kullback-Leibler divergence similarity measure. Meanwhile, no-building indicator was generated by vegetation and shadow classifications and objectbased building extraction was implemented based on shape features, which contributed to improving the change detection results. Based on the supervised classification by combing height, spectral and shape information, Qin et al. (2015) presented a 3D building change detection approach using multitemporal stereo images. A synergic mean-shift segmentation method was applied on the orthophotos with the constraints of the DSM to derive segments with homogenous spectrum and height, which were then classified with a hybrid decision tree

\footnotetext{
* Corresponding author
} 
and SVM approach. To identify the change status of each building, an initial change indicator (CI) was computed and an adaptive updating strategy based on segment overlapping was proposed. These methods need dense image matching technology to generate DSM or DEM which usually contains many noises caused by building shadow and perspective projection. Consequently, large amount of post-processing work is indispensable for removing noises to obtain reasonable building change map.

2. DSM is generated by LiDAR data and building map is obtained by the comparison between DSM and existing building database or between DSM and building models. Matikainen et al. (2004) performed building change detection using LiDAR data, aerial images and building database. DSM generated by LiDAR data was firstly segmented, then DSM was classified using height, spectral and textual information to obtain building areas, finally building change map was achieved by the comparison between extracted building areas and building database. Vosselman et al. (2005) carried out building change detection using LiDAR data and existing building database. In the paper, DSM generated by LiDAR data was firstly segmented and classified to obtain building areas, then building map was achieved by the boundary comparison between extracted buildings and buildings in the database. Rottensteiner (2008) presented a building change detection method using LiDAR data and high resolution satellite images. D-S theory fusion, topological clarification and change areas classification were performed on DSM generated by LiDAR data for obtaining building areas, after which building map was generated by the comparison between building areas and building database. Chen et al. (2010) proposed a method of comparing LiDAR data and existing building models by double-thresholds strategy, and building change detection accuracy was further improved using spectral information from aerial images. Liu et al. (2014) proposed an automated building change detection method using UltraCamD images and existing CAD data. Edge features of buildings were extracted from images and silhouettes of buildings were extracted from CAD models. Then Hausdorff matching methodology was employed to calculate the similarity, which was used to detect the collapsed and removed buildings. Qin (2014) conducted a building change detection study with 3D city models and Very High-resolution(VHR) images. Firstly, 3D building models were projected onto a raster grid, and DSM was extracted by stereo imagery with SGM method. Secondly, a multi-channel change indicator was extracted between the 3D models and stereo images, which was then clustered and analyzed with Self-organizing Maps(SOM) and Markov Random Field(MRF). Finally, buildings were extracted by combining multi-spectral images and DSM, then building change types were determined. However, all of the above methods involve the assistance of building database or building models, resulting in the complex operations of converting vector map into grid map and the co-registration between grid map and DSM. These operations influence the accuracy, speed and automation of building change detection to a certain degree. In addition to that, building database or building models are hard to be achieved in most cases, especially in rural areas, which reduces the generality of the methods.

Based on the analysis above, we propose a building change detection method combining LiDAR data and ortho images, which well combines the advantages of different sources of data for the purpose of quantitative analysis of 3D building change detection. Height change information is achieved from DSM generated by LiDAR data. DSM change areas are then projected onto the ortho images through thematic segmentation. False building change areas are excluded by geometric information from DSM and spectral and textual information from ortho images. Finally, the correctness and completeness are computed using manually digitized reference building map to evaluate the accuracy of the proposed method. The correctness and feasibility of the proposed method is verified by the 3D building change detection experiment of the test areas.

This paper is organized as follows. In Section 2, we describe the study areas and data sources. The proposed methodology is illustrated in detail in Section 3. The result of the experiment is discussed in Section 4. Finally, Section 5 draws the conclusion.

\section{STUDY AREAS AND DATA SOURCES}

\subsection{Aerial Ortho Images and LiDAR Data}

In order to demonstrate the capability of the proposed building change detection method, two periods of aerial ortho images and corresponding LiDAR data, acquired on different occasions over suburb areas of Guangzhou City China, are used for the experiments. The test site covers an area of $1 \mathrm{~km}^{2}$, including water areas, large industrial areas, dense residential areas, network of main and local roads, open land and grass areas as well as trees. Moreover, LiDAR data was collected by Trimble H68 system in September 2011 and August 2012 with the point density of $4 \mathrm{pts} / m^{2}$ to $6 \mathrm{pts} / \mathrm{m}^{2}$, the size of aerial ortho images is 5000 pixel $\times 5000$ pixel with ground sample distance (GSD) of $0.2 \mathrm{~m}$. The DSMs generated by LiDAR data and aerial ortho images are shown in figure 1 .

\subsection{Reference Data}

In order to accurately evaluate the performance of the proposed change detection method, building changes were manually interpreted and digitized. The reference data is a three-class thematic image, typically divided into the following three categories: 'Newly-built', 'Heightened' and 'Demolished'.

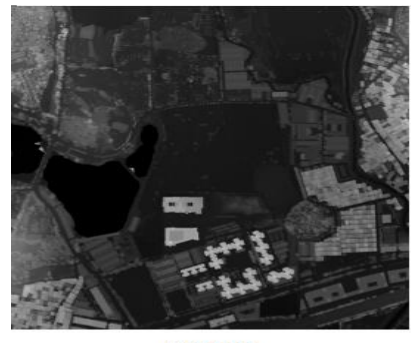

(a)DSM1

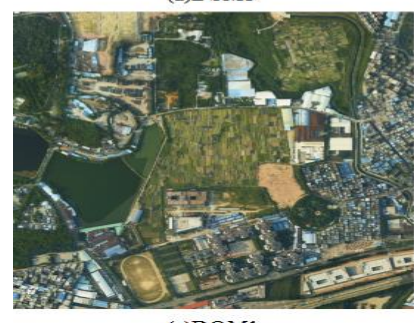

(c)

Figure 1. Two periods of DSMs and DOMs

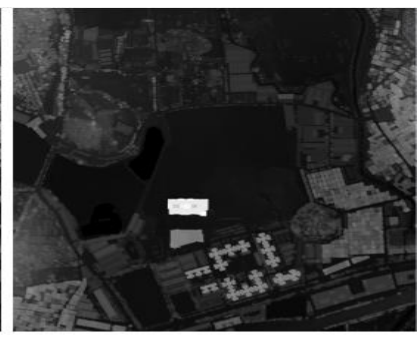

(b)DSM2

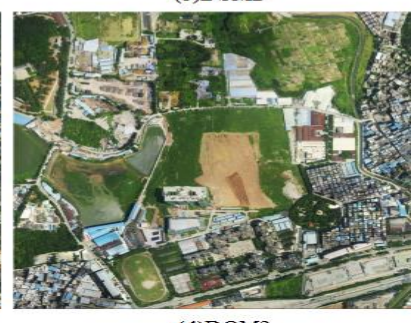

(d)DOM2 


\section{METHODOLOGY}

Height change reflects the main change of buildings and elevation information is an important index for extracting building change information (Stal et al., 2013). High precision DSM can be generated by LiDAR data and building height change can be obtained through DSM differencing, then new or demolished buildings can be detected (Alobeid et at., 2011). However, the lack of textual information and uneven distribution of LiDAR point density make it hard to remove false changes caused by trees and terrain. On the contrary, aerial ortho images are rich in spectral and textual information. Specifically, green band ratio and Normalized Difference Vegetation Index (NDVI) are of great significance for distinguishing trees against buildings (Rottensteiner et al., 2007). Therefore, the combining of LiDAR data and aerial ortho images could achieve the supplementary advantages of different data sources and thus improve the building change detection accuracy. In this paper, a novel method of building change detection by combining LiDAR data and aerial ortho images is proposed as illustrated in figure 2 .

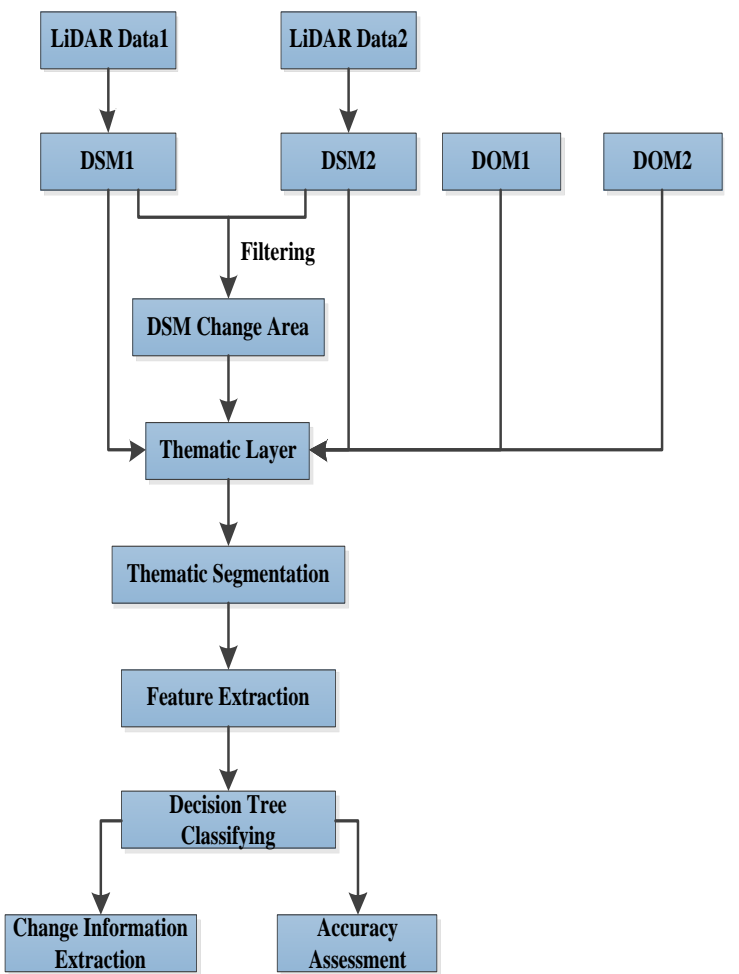

Figure 2. The flow chart of 3D building change detection

The proposed approach consists of the following steps:

1. DSM change area extraction. Two periods of DSMs are generated after the operation of filtering and interpolating of two periods of LiDAR data. Then DSM change areas are obtained by DSM differencing, height filtering, height textual filtering, morphological filtering and area filtering.

2. Feature Extraction of DSM Change Areas. Shape file of DSM change areas is generated through eCognition software (Li et al., 2014), which is used for thematic segmentation of the two periods of DSMs and DOMs. Then height features, spectral features and textual features are extracted from the segmented DSMs and DOMs.
3. Building change segments identification and regularization. The optimal classification feature combination is determined by the optimal classification distance, after which decision tree method is applied to classify the DSM change areas for obtaining building change areas. Building contours are then regularized for accurate change information extraction.

4. Change information extraction and accuracy assessment. Elevation and area change information of building change areas are calculated and the corresponding change types are categorized. Moreover, the completeness and correctness are calculated to evaluate the accuracy of the proposed building change detection method.

\subsection{DSM Change Areas Extraction}

Building corner features are extracted using two periods of LiDAR data followed by 3D similarity transformation to implement the co-registration of two periods of LiDAR data (Zhang et al., 2012). Then DSMs with 16 bits grey quantization and $0.2 \mathrm{~m}$ sample intervals are generated through interpolation, after which DSM differencing is implemented to obtain the differential DSM (dDSM). However, due to the noises caused by terrain and non-building areas such as trees, height filtering is necessary to remove the noises out. Considering the fact that building areas are usually higher than the noisy areas and the average height of one-story building is about $2.5 \mathrm{~m}$, we set the threshold to $2.5 \mathrm{~m}$ for height filtering with the Equation (1) shown as follows.

$$
\operatorname{Diff}(i, j)=\left\{\begin{array}{c}
65535 \quad \text { if } x_{2}(i, j)-x_{1}(i, j)>T_{h} \\
0 \quad \text { if }\left|x_{2}(i, j)-x_{1}(i, j)\right|<T_{h} \\
x_{1}(i, j)-x_{2}(i, j) \quad \text { if } x_{2}(i, j)-x_{1}(i, j)<-T_{h}
\end{array}\right.
$$

Where $\operatorname{Diff}(i, j)$ is the grey value after differencing, $x_{1}(i, j)$ is the grey value of epoch $T_{1}, x_{2}(i, j)$ is the grey value of epoch $T_{2}, T_{h}$ is the height threshold, we set $T_{h}=2.5 \mathrm{~m}$.

DSM after height filtering is shown in figure 3(a), where the white areas represent the positive change areas while the grey areas correspond to the negative change areas. We can see that the non-building noises have been removed to a certain degree after height filtering. However, a large amount of non-building change areas still exist as shown in figure 3(a), where Label 1 area represents trees while Label 2 area represents building edges, either of which is impossible to be removed through height filtering. Height texture, which represents local height change of target objects, is of great significance to the distinguishing between buildings and non-buildings such as trees and roads (Tiwari and Pande, 2008). Generally, local height change of buildings is small, while local height change of trees and building edges is large, which is an important indicator in distinguishing buildings against non-buildings. In this paper height texture entropy is selected as the textual feature value as shown in Equation (2).

$$
E(i, j)=-\sum_{x=0} \sum_{y=0} p(x, y) \operatorname{In}(x, y)
$$

Where $E(i, j)$ is the textual entropy, $p(x, y)$ is the probability of grey level pairs $(x, y)$. 
The height texture filtering formula is defined in Equation (3) and the filtering result is shown in figure 3(b).

$$
\operatorname{Diff}(i, j)=\left\{\begin{array}{lr}
\operatorname{Diff}(i, j), E(i, j) \leq T_{e} \\
0 \quad, E(i, j) \leq T_{e}
\end{array}\right.
$$

Where $\operatorname{Diff}(i, j)$ is the grey value after height filtering, $T_{e}$ is the threshold of height texture entropy.

As shown in figure 3(b), most areas with large local height changes such as trees and building edges are removed. Meanwhile, large areas with large local height changes will be broken into small areas, which is very useful for further filtering process. However, non-building change noises caused by random reflection of building edges and different flight parameters when collecting LiDAR data still exist widely (Vu et al., 2004). Morphological opening operation has the ability of smoothing the image contours and disconnecting the narrow connections. Hence, morphological opening operation is implemented to remove the noises above. In our test the window size of the morphological filtering is set to be $21 \times 21$. The filtering result is shown in figure 3(c). As shown in figure $3(\mathrm{c})$, most narrow edge areas and broken areas are removed. Nevertheless, many small isolated change areas still exist, which is mainly caused by small objects on the roof, low-rise buildings and building accessory structures such as chimneys and balconies. In general, building change detection focuses on large buildings, therefore it's necessary to remove small building change areas using area threshold. In our test we set the area threshold to $80 \mathrm{~m}^{2}$ and obtain the final binary dDSM as shown in figure 3(d). We can see that most non-building noises are removed and DSM change areas with clear background are generated after height filtering, height texture filtering, morphological filtering and area filtering.
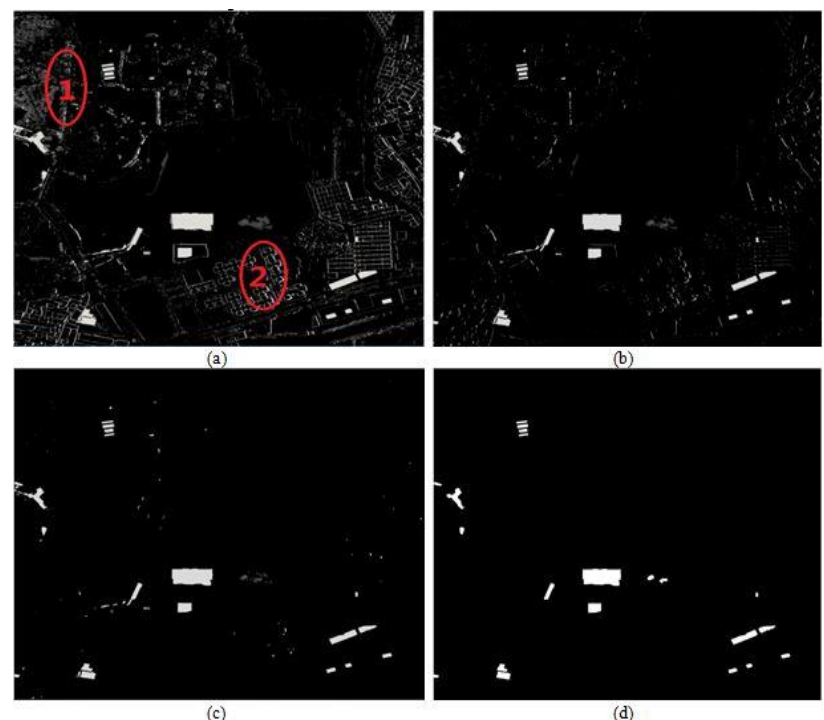

Figure 3. Filtering result of dDSM. (a) dDSM after height filtering, (b) dDSM after height texture filtering, (c) dDSM after morphological filtering, (d) dDSM after area filtering

\subsection{Feature Extraction of DSM Change Areas}

Firstly, shape file of DSM change areas is generated by eCognition software, which is used as the thematic layer for the segmentation of DSM and DOM. Secondly, DSM change areas are classified into ground areas and non-ground areas based on the inherent classification of the LiDAR data. Finally, object features of DSM and DOM are extracted. In object-based classification, object features include spectrum, shape, texture and context semantics, each of which contains several specific descriptors that have significant contribution to the classification (Zhang et al., 2009). A certain number of representative samples distributing evenly are selected for the selection of the best description features. In feature space, the best description features are determined by the best classification distance between the samples of two classes, and the formula is shown as follows (Wang et al., 2009):

$$
\mathrm{D}=\sqrt{\sum_{\mathrm{f}_{\mathrm{i}}}\left(\frac{\mathrm{v}_{\mathrm{f}_{\mathrm{i}}}{ }^{(\mathrm{s})}-\mathrm{v}_{\mathrm{f}_{\mathrm{i}}}{ }^{(\mathrm{o})}}{\sigma_{\mathrm{f}_{\mathrm{i}}}}\right)^{2}}
$$

Where $\mathrm{f}_{\mathrm{i}}$ represents the $i$ th feature in the feature space, D represents the distance between training samples object $s$ and classification image object $\boldsymbol{o}, \mathrm{V}_{\mathrm{f}_{\mathrm{i}}}{ }^{(\mathrm{s})}$ represents the feature value of training sample $s, \mathrm{v}_{\mathrm{f}_{\mathrm{i}}}{ }^{\left({ }^{\circ}\right)}$ is the feature value of classification object $\boldsymbol{o}, \sigma_{\mathrm{f}_{\mathrm{i}}}$ represents the standard deviation of all the image objects in the feature space. The features used are listed in Table 1.

\begin{tabular}{cc}
\hline $\begin{array}{c}\text { Feature class } \\
\text { (Data Source) }\end{array}$ & Attributes of segments \\
\hline $\begin{array}{c}\text { Geometry } \\
\text { (DSM) }\end{array}$ & Mean Height, Standard deviation of Height \\
$\begin{array}{c}\text { Spectrum } \\
\text { (DOM) }\end{array}$ & Ratio of Green Band, Mean, Standard \\
Texture & GLCM Homogeneity, GLCM Contrast, \\
$($ DOM $)$ & GLCM Dissimilarity, GLCM Entropy \\
Shape & Asymmetry, Compactness, Density, Main \\
(DOM) & direction, Rectangular Fit, Roundness, \\
& Shape Index \\
\hline Table 1. Features used in the DSM and DOM segments
\end{tabular}

Through calculation we come to the fact that the best geometric description feature of DSM is Mean Height (MH), and the best spectral, textual and shape description features are Ratio of Green Band (RG), GLCM (Grey-Level Co-occurrence Matrix) Homogeneity (GH), Asymmetry (AS) respectively. Moreover, the classification distances of two dimensional features from the four features are calculated to select the best feature combination as shown in figure 4 . We can see that $\mathrm{RG}+\mathrm{MH}$ are the optimal feature combination.

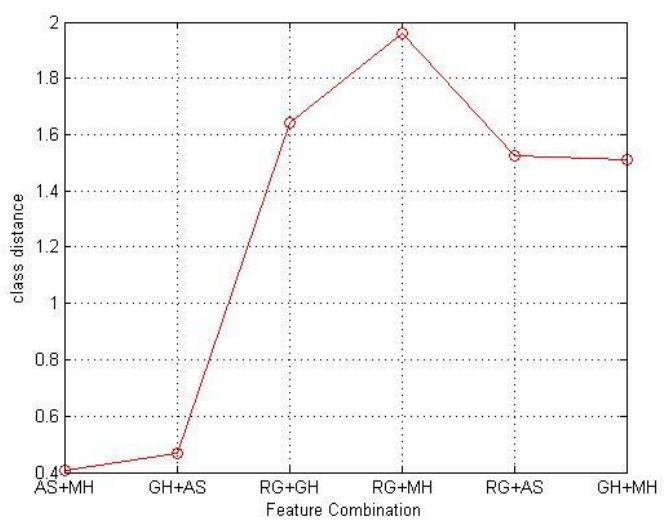

Figure 4. Class distance of different feature combination 


\subsection{Decision Tree Classification}

Decision tree classification is a non-parametric classification method, which relies on no prior statistical hypothesis and possesses good applicability to new data. Moreover, it's simple, accurate and fast, enjoying wide use in classifying remote sensing images. Within decision tree, each bifurcation point represents a decision condition, and the two leaf nodes at each bifurcation point represent the type of meeting the condition or not. In this paper, Gini index, which is the measure of nodes' impurity, is chosen as the splitting criterion for each node. It's defined as follow (Friedl, Brodley et al., 1997):

$$
\operatorname{impurity}(t)=\sum_{i \neq j} p(i \mid t) p(\mathrm{j} \mid t)
$$

Where $t$ represents a node, $p(i \mid t)$ and $p(\mathrm{j} \mid t)$ are the probabilities that feature vector $x_{n}$ belongs to class $i$ and class $j$ respectively. In decision tree classification, each node will search the split that reduces the node impurity the most. A node has to contain at least 10 training segments to be split. In our test, a certain number of sample areas representing different object classes and distributing evenly are selected, followed by the calculation of RG and MH of each sample areas. Then decision tree is constructed by the training samples and tree pruning is implemented for reducing the fluctuation caused by training dataset noise and the overfitting due to the over-complication of the decision tree. Training data and 10-fold cross-validation are used to estimate the best level of pruning by computing the costs of subtrees. The costs are based on the misclassifications produced by the trees. Finally DSM change areas are classified by the decision tree and the classification result is shown in figure 5. In order to describe each DSM change areas more clearly, a series of numerical symbols are assigned respectively. Moreover, different colours are used for labelling classification result of each DSM change areas. As seen from figure 5, DSM change areas are classified into four categories of 'Ground', 'Building', 'Tree' and 'Water'. Building change consists of 'from Ground to Building' (corresponding labels are $1,2,3,4,5,6,11,14,15,16,17,19)$, 'from Building to Building'(corresponding labels are 8,12,13,18,21), and 'from Building to Ground'(corresponding label is 20), while false change involves 'from Water to Ground'(corresponding label is 7) and 'from Ground to Tree'(corresponding labels are 9,10).

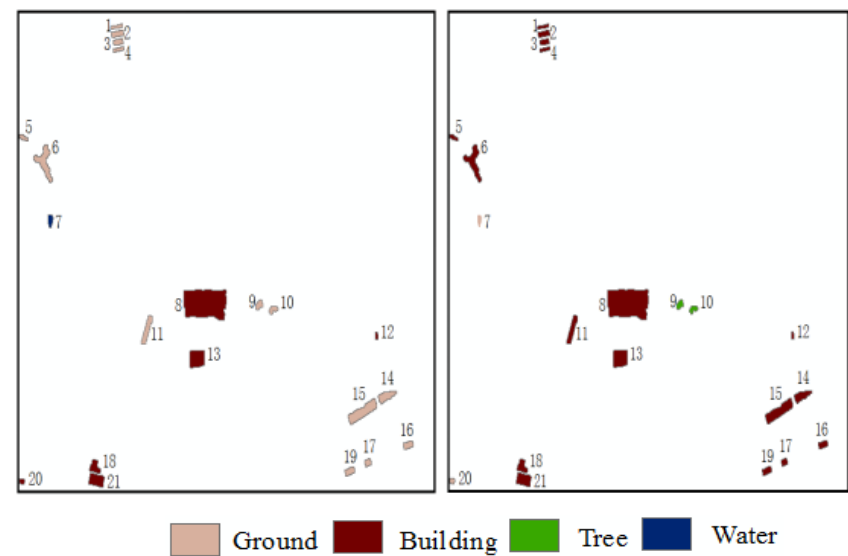

Figure 5. Decision tree classification result of two periods of datasets

\subsection{Regularization of Building Change Areas}

After four filtering operations during the process of obtaining DSM change areas, building edges become distorted and unsharp, which are quite different from the true contours of the buildings. In addition to that, the statistics of $3 \mathrm{D}$ building change information which includes area change information and height change information is significantly influenced. Hence, it's important to regularize the contours of building change areas. To the end, contour tracking of building change areas is implemented, followed by the calculation of the minimum enclosing rectangle and the computation of the ratio between the area of the minimum enclosing rectangle and the area of the building contour. If the ratio exceeds the defined threshold, we consider that the building is not a regular rectangle area and the Douglas-Peucker method is applied for building contour approximation, otherwise the minimum enclosing rectangle is chosen as the building contour. Two different types of building contour regularization results are shown in Figure 6. We can see that both rectangular building contour and non-rectangular building contour are more closer to true building contour after regularization, which compensates the unsharping effect caused by filtering.
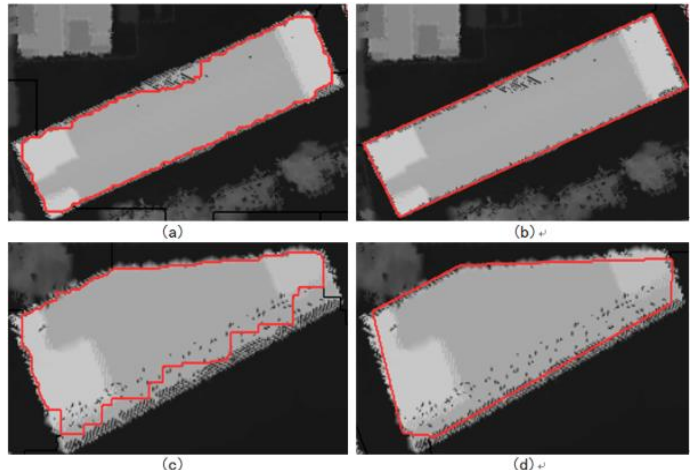

Figure 6. Building contour regularization result. (a) and (b) are rectangle building areas before and after regularization, (c) and (d) are non-rectangular building areas before and after regularization

\section{RESULTS AND DISCUSSIONS}

Building change areas are obtained using the proposed approach, meanwhile, 3D building change information are calculated. In this paper, height change information is obtained by computing the difference of average height value between pre-change areas and post-change areas, while footprint area change information is obtained by the statistic of building change area pixels after regularization.

The 3D building change detection result is shown in Table 2, where 'Change area ID' refers to the labels of DSM change areas as shown in figure 5, 'Change content' represents the classification results of two periods of DSM change areas, 'Height change' refers to the elevation difference of two periods of building change areas, 'Area change' indicates the area difference of two periods of building change areas, 'Change type' refers to the change category of two periods of DSM change areas.

As can be seen from Table 2, within the 21 DSM change areas, 18 DSM change areas are building change areas while the rest are false change areas. And the 18 building change areas are composed of 12 newly-built buildings and 6 heightened 
buildings. The range of building elevation change is from $5 \mathrm{~m}$ to $30 \mathrm{~m}$, while the range of building area change is between $160 \mathrm{~m}^{2}$ and $1000 \mathrm{~m}^{2}$. What's more, newly-built buildings are buildings changed from ground to building which contain both height change and footprint area change, while heightened buildings are buildings changed from lower buildings to higher buildings caused by height change alone and can only be detected by height information. The $3 \mathrm{D}$ building change information and change type provided by Table 2 is quite important to the accurate and comprehensive analysis of building change, especially for urban invalid building monitoring and post-disaster building demolishment assessment. For the purpose of the vivid description of the building change and the storing of building change detection result in the vector format, building change map is generated as shown in Figure 7.

\begin{tabular}{clccc}
\hline Change area ID & Change content & $\begin{array}{c}\text { Height change } \\
(\mathrm{m})\end{array}$ & $\begin{array}{c}\text { Area change } \\
\left(\mathrm{m}^{2}\right)\end{array}$ & Change type \\
\hline 1 & Ground $\rightarrow$ Building & 5.67 & 293.24 & Newly-built \\
2 & Ground $\rightarrow$ Building & 5.42 & 191.28 & Newly-built \\
3 & Ground $\rightarrow$ Building & 5.71 & 241.84 & Newly-built \\
4 & Ground $\rightarrow$ Building & 5.75 & 165.08 & Newly-built \\
5 & Ground $\rightarrow$ Building & 6.67 & 155.92 & Newly-built \\
6 & Ground $\rightarrow$ Building & 12.71 & 920.08 & Newly-built \\
7 & Water $\rightarrow$ Ground & --- & --- & False change \\
8 & Building $\rightarrow$ Building & 30.45 & 0 & Heighted \\
9 & Ground $\rightarrow$ Tree & --- & --- & False change \\
10 & Ground $\rightarrow$ Tree & --- & False change \\
11 & Ground $\rightarrow$ Building & 7.70 & 690.28 & Newly-built \\
12 & Building $\rightarrow$ Building & 6.78 & 0 & Heighted \\
13 & Building $\rightarrow$ Building & 5.38 & 0 & Heighted \\
14 & Ground $\rightarrow$ Building & 20.03 & 564.68 & Newly-built \\
15 & Ground $\rightarrow$ Building & 19.28 & 1342.32 & Newly-built \\
16 & Ground $\rightarrow$ Building & 10.62 & 281.52 & Newly-built \\
17 & Ground $\rightarrow$ Building & 10.62 & 183.08 & Newly-built \\
18 & Building $\rightarrow$ Building & 6.59 & 0 & Heighted \\
19 & Ground $\rightarrow$ Building & 10.58 & 285.6 & Newly-built \\
20 & Building $\rightarrow$ Ground & -16.57 & 0 & Demolished \\
21 & Building $\rightarrow$ Building & 5.43 & 0 & Heighted \\
\hline
\end{tabular}

Table 2. Statistic of 3D building change detection

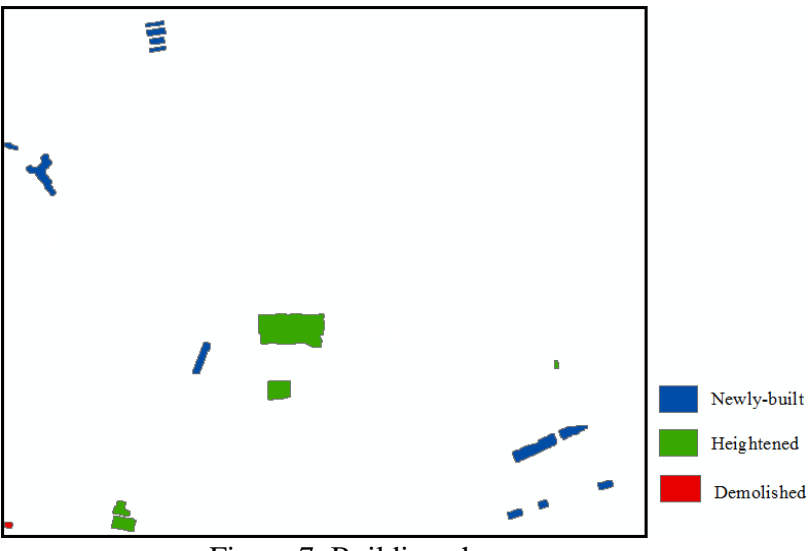

Figure 7. Building change map

In order to evaluate the accuracy of the proposed building change detection method, the completeness and correctness of building change detection are introduced as the evaluation criteria, which are defined as follows:

$$
\begin{aligned}
& \text { Completeness }=\frac{T P}{T P+F N} \\
& \text { Correctness }=\frac{T P}{T P+F P}
\end{aligned}
$$

Where TP is the number of true positives which represent the new buildings that have been detected correctly, FP is the number of false positives which represent the buildings that the proposed method detects as changed but are actually unchanged, and FN is the number of false negatives which represent the buildings that the proposed method detects as unchanged, but that nevertheless have changed.

The threshold of building size is an important factor in obtaining the final building change map, in order to discuss the influence of the threshold of building size on building change detection accuracy, a series of area thresholds are selected and the corresponding completeness and correctness of building change detection are calculated and shown in Figure 8.

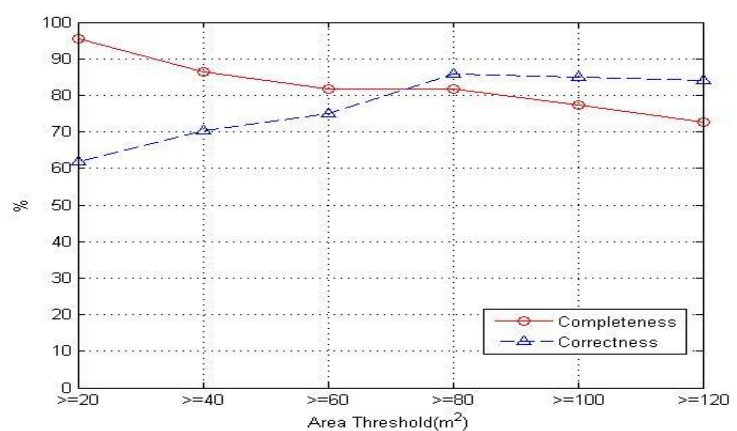

Figure 8 . The influence of area threshold on building change detection accuracy.

As can be seen from figure 8 , the completeness of building change detection approaches to $95 \%$ and the correctness approaches to $61 \%$ when the area threshold is set to be $20 \mathrm{~m}^{2}$, 
in which case the correctness of building change detection is mainly decreased by small size building appendages. Along with the increase of area threshold, small size building appendages are removed, leading to the decrease of the completeness of building change detection and the increase of the correctness of building change detection. When the area threshold is set to be $80 \mathrm{~m}^{2}$, the completeness of building change detection is about $85 \%$, while the correctness of building change detection reaches the highest value of $81 \%$, which shows that the building size in the test area is mainly larger than $80 \mathrm{~m}^{2}$. When the area threshold is larger, the completeness of building change detection decrease sharply while there is a small decrease of the correctness of building change detection, reflecting the fact that only a small number of large nonbuilding change areas exist which cannot be removed by area filtering.

To evaluate the advantages of the proposed method, the completeness and correctness of building change detection are compared between the proposed approach and the other two building change detection methods using DOM or LiDAR data only. The result is shown in Table 3. As can be seen, both the completeness and correctness of the proposed approach increase about $10 \%$ when compared with results using only DOM, which verifies the importance of combing LiDAR data and DOM for building change detection. Moreover, we can see that when using LiDAR data only, both the completeness and correctness are about $6 \%$ higher than that using DOM only, which indicates the importance of height information in building change detection.

\begin{tabular}{ccc}
\hline Method & $\begin{array}{c}\text { Completeness } \\
(\%)\end{array}$ & $\begin{array}{c}\text { Correctness } \\
(\%)\end{array}$ \\
\hline DOM & 71.2 & 76.3 \\
LiDAR data & 77.5 & 82.4 \\
DOM \& & $\mathbf{8 1 . 8}$ & $\mathbf{8 5 . 7}$ \\
LiDAR data & &
\end{tabular}

Table 3. Completeness and correctness of the three methods

\section{CONCLUSIONS}

Building change detection is an important issue in urban planning and disaster assessment. In this paper, a novel building change detection method by combining LiDAR data and DOM is proposed, which relies on no building databases and possesses the complementary advantages of pixel-level and object-level change detection. The advantages of pixel-level change detection is presented by DSM differencing to obtain candidate building change areas while the advantages of objectlevel change detection is reflected by thematic segmentation which implements the projection of DSM change areas onto original DSMs and DOMs followed by decision tree classification using object features extracted from both DSMs and DOMs. The experiment results demonstrate the validness and feasibility of the proposed approach. Compared with the traditional building change detection methods, the proposed method can determine the building change type and quantitatively extract $3 \mathrm{D}$ building change information which is of great significance for the comprehensive and accurate analysis of building change. It's worth to point out that the presented building change detection approach is characterized by LiDAR data with DOMs for auxiliary analysis, how to implement a deeper level of fusion of the two sources of data for more accurate building change detection is our further work.

\section{ACKNOWLEDGEMENTS}

This work was supported in part by the National Natural Science Foundation of China under Grant 41322010 and 41571434

\section{REFERENCES}

Adar, S., Shkolnisky, Y., Dor, E. B., 2014. A new approach for thresholding spectral change detection using multispectral and hyperspectral image data, a case study over Sokolov, Czech republic. International Journal of Remote Sensing, 35(4), pp. 1563-1584.

Alobeid, A., Jocobsen, K., Heipke, C., Rajhi, M. A., 2011. Building Monitoring with Differential DSMs, ISPRSInternational Archives of the Photogrammetry, Remote Sensing and Spatial Information Sciences, 3819, pp. 7-16.

Bovolo, F., Bruzzone, L., 2007. A split-based approach to unsupervised change detection in large-size multitemporal images: application to tsunami-damage assessment. IEEE Transactions on Geoscience and Remote Sensing, 45(6), pp. 1658-1670.

Cao, G., Li, Y., Shang, Y ., 2014. Automatic change detection in high-resolution remote sensing images by means of level set evolution and support vector machine classification. International Journal of Remote Sensing, 35(16), pp. 62556270.

Champion, N., 2007. 2D building change detection from high resolution aerial images and correlation digital surface models. International Archives of Photogrammetry, Remote Sensing and Spatial Information Sciences, 36(3/W49A), pp. 197-2002.

Chen, L. C., Lin, L. J., 2010. Detection of building changes from aerial images and light detection and ranging (LIDAR) data. Journal of Applied Remote Sensing, 4(12), pp. 2785-2802.

Franklin, S. E., Ahmed, O. S., Wulder, M. A., White, J. C., Hermosilla, T., Coops, N. C., 2015. Large area mapping of annual land cover dynamics using multi-temporal change detection and classification of Landsat time series data. Canadian Journal of Remote Sensing, pp. 293-314.

Friedl, M. A., Brodley, C. E., 1997. Decision tree classification of land cover from remotely sensed data. Remote sensing of environment, 61(3), pp. 399-409.

Gamba, P., Houshmand, B., 2002. Joint analysis of SAR, LIDAR and aerial imagery for simultaneous extraction of land cover, DTM and 3D shape of buildings. International Journal of Remote Sensing, 23(20), pp. 4439-4450.

Huo, C., Zhou, Z., Lu, H., Pan, C., Chen, K., 2010. Fast ObjectLevel Change Detection for VHR Images. IEEE Geoscience \& Remote Sensing Letters, 7(1), pp. 118-122.

Jung, F., 2004. Detecting building changes from multitemporal aerial stereopairs. ISPRS Journal of Photogrammetry and Remote Sensing, 58(3), pp. 187-201. 
Knudsen, T., Olsen, B. P., 2003. Automated change detection for updates of digital map databases. Photogrammetric Engineering \& Remote Sensing, 69(11), pp. 1289-1296.

Li, L., Gong, Y., Li, X., Wang, K., 2014. Change detection based on similarity measurement of object histogram using high-resolution remote sensing imagery. Journal of Remote Sensing, 18(1), pp. 139-153.

Liu, Z., Gong, P., Shi, P., Chen, H., Zhu, L., Sasagawa, T. 2014 Automated building change detection using UltraCamD images and existing CAD data. International Journal of Remote Sensing, 31(6), pp. 1505-1517.

Matikainen, L., Hyyppä, J., Kaartinen, H., 2004. Automatic detection of changes from laser scanner and aerial image data for updating building maps. International Archives of Photogrammetry, Remote sensing and Spatial Information Sciences, 35(B2), pp. 434-439.

Murakami, H., 1999. Change Detection of Buildings Using An Airborne Laser Scanner. ISPRS Journal of Photogrammetry \& Remote Sensing, 54(2), pp. 148-152.

Nutini, F., Boschetti, M., Brivio, P. A., Bocchi, S., Antoninetti, M., 2013. Land use and land-cover change detection in a semiarid area of Niger using multi-temporal analysis of Landsat images. International Journal of Remote Sensing, 34(13), pp. 4769-4790.

Niemeyer, I., Marpu, P. R., Nussbaum, S., 2007. Change detection using the object features. Geoscience and Remote Sensing Symposium, IGARSS 2007. IEEE International, pp. 2374-2377.

Qin, R., Huang, X., Gruen, A., Schmitt, G., 2015. Object-based 3-D building change detection on multi-temporal stereo images. IEEE Journal of Selected Topics in Applied Earth Observations \& Remote Sensing, 8(5), pp. 1-13.

Qin, R., 2014. Change detection on LOD 2 building models with very high resolution spaceborne stereo imagery. ISPRS Journal of Photogrammetry \& Remote Sensing, 96(11), pp. 179-192.

Rottensteiner, F., Trinder, J., Clode, S., Kubik, K., 2007. Building detection by fusion of airborne laser scanner data and multi-spectral images:Performance evaluation and sensitivity analysis. ISPRS Journal of Photogrammetry \& Remote Sensing, 62(2), pp. 135-149.

Singh, A., 1989. Digital Change Detection Techniques Using Remotely Sensed Data. International Journal of Remote Sensing, 10(6), pp. 989-1003.

Stal, C., Tack, F., De Maeyer, P., De Wulf, A., Goossens, R., 2013. Airborne photogrammetry and lidar for DSM extraction and 3D change detection over an urban area-a comparative study. International Journal of Remote Sensing, 34(4), pp. 1087-1110.

Tian, J., Cui, S., Reinartz, P., 2014. Building change detection based on satellite stereo imagery and digital surface models. IEEE Transactions on Geoscience \& Remote Sensing, 52(1), pp. 406-417.
Tiwari, P. S., Pande, H., 2008. Use of laser range and height texture cues for building identification. Journal of the Indian Society of Remote Sensing, 36(3), pp. 227-234.

Vögtle, T., Steinle, E., 2004. Detection and recognition of changes in building geometry derived from multitemporal laser scanning data. International Archives of Photogrammetry and Remote Sensing, 35(B2), pp. 428-433.

Vosselman, G., Kessels, P., Gorte, B., 2005. The utilisation of airborne laser scanning for mapping. International Journal of Applied Earth Observation and Geoinformation, 6(3), pp. 177186.

Vu, T. T., Matsuoka, M., Yamazaki, F., 2004. Lidar-based change detection of buildings in dense urban areas. Geoscience and Remote Sensing Symposium, IGARSS'04. Proceedings. 2004 IEEE International, 5, pp. 3413-3416.

Wang, B., Choi, S., Byun, Y., Lee, S., Choi, J., 2015. ObjectBased Change Detection of Very High Resolution Satellite Imagery Using the Cross-Sharpening of Multitemporal Data. IEEE Geoscience \& Remote Sensing Letters, 12, pp. 1151-1155.

Wang, W., Zhao, Z., Zhu, H., 2009. Object-oriented multifeature fusion change detection method for high resolution remote sensing image. Geoinformatics, 17th International Conference on IEEE, pp. 1-6.

Zhang, X., Fong, X., Hong, J., 2009. Feature space optimizing based on object classification. Journal of Remote Sensing, 13(4), pp. 664-669.

Zhang, Y., Xiong, X., 2012. Automatic registration of urban aerial imagery with airborne LiDAR data. Journal of Remote Sensing, 16(3), pp. 579-595.

Zhu, Z., Woodcock, C. E., 2014. Continuous change detection and classification of land cover using all available Landsat data. Remote Sensing of Environment, 144(1), pp. 152-171. 